\title{
Determination of Pyrazinamide in Human Plasma Samples Containing Fixed Dose Combination Molecules by using Liquid Chromatography Tandem Mass Spectrometry
}

Chaitanya Krishna $A^{1 *}$, Saravanan $\mathbf{R S}^{2}$, Jeevanantham $\mathbf{S}^{2}$, Vignesh $\mathbf{R}^{2}$ and Karthik $\mathrm{P}^{2}$

${ }^{1}$ Study Director, Bombay Bioresearch Centre, Govandi-400043, Mumbai, India

${ }^{2}$ Department of Bioanalaytical, Bombay Bioresearch Centre, Govandi-400043, Mumbai, India

\begin{abstract}
A rapid, simple, sensitive and compatible liquid chromatography tandem mass spectrometric method has been developed and validated for the estimation of Pyrazinamide. Glipizide was used as an internal standard. Detection was performed using TSQ Quantum Discovery max mass spectrometer with ESI source in positive polarity. The detection transition for Pyrazinamide is $124.100 \rightarrow 79.160$ and for Glipizide is $446.200 \rightarrow 321.200$. Chromatographic separation of analyte and internal standard were carried out using a reverse phase column, Hypersil, Gold, $4.6 \mathrm{X}$ $50 \mathrm{~mm}, 5 \mu$ at a flow rate of $0.400 \mathrm{~mL} / \mathrm{min}$. Mobile Phase is composed of Methanol: $0.1 \%$ FA in $10 \mathrm{mM}$ Ammonium Formate $(90: 10) \mathrm{v} / \mathrm{v}$. Extracted by Solid Phase Extraction with a sample volume of $200 \mu \mathrm{L}$ plasma. The assay of Pyrazinamide is linear over the range of $0.935 \mu \mathrm{g} / \mathrm{mL}$ to $60.408 \mu \mathrm{g} / \mathrm{mL}$ with a precision of $<9.86 \%$. Mean extraction recovery for Pyrazinamide and Glipizide were more than $61 \%$. Samples are stable at room temperature for 6 hrs, processed samples were stable at least for $28 \mathrm{hrs}$ and also stable at three freeze-thaw cycles.
\end{abstract}

Keywords: Pyrazinamide; LC-MS/MS; Validation; Human Plasma

\section{Introduction}

Pyrazinamide is a pyrazine carboxyl amide; Pyrazinecarboxamide with a molecular formula $\mathrm{C}_{5} \mathrm{H}_{5} \mathrm{~N}_{3} \mathrm{O}$ and molecular weight $123.11 \mathrm{~g} / \mathrm{mol}$ [1], pKa of 0.5 Pyrazinamide, the pyrazine analogue of nicotinamide, is an antituberculous agent. It may be used as a bacteriostatic or bacteriocidal against Mycobacterium tuberculosis depending on the concentration of drug attained at the site of infection.

There are several studies that were performed on Fixed Dose Combinations (FDC) medicines and on individual dosage forms. Though, comparative studies between both FDC and individual drugs are available, the response of patients to the treatment depends on the resistance to the drugs. Recommended doses of first line antituberculosis drugs for adults were Isoniazid 8-12mg/kg/dose, Rifampicin 8-12mg/ $\mathrm{kg} / \mathrm{dose}$, Pyrazinamide $20-30 \mathrm{mg} / \mathrm{kg} / \mathrm{dose}$ and Ethambutol $15-20 \mathrm{mg} /$ $\mathrm{kg} / \mathrm{dose}[2]$.

As per the reported literatures, for the determination of Pyrazinamide in human plasma, no articles has been found as an individual or combined method with more than a recovery of $50 \%$. More over, these methods should also be compatible for the determination of Pyrazinamide in human plasma containing ascorbic acid which is required for the stability of Rifampicin $[3,4]$ especially, in case of samples containing fixed dose combination formulation. Hence, authors propose a method that is developed and validated to overcome the difficulties in achieving the required extraction recoveries that will be useful for carrying out analysis of samples containing multiple drugs.

\section{Experimental}

\section{Materials and reagents}

Pyrazinamide is purchased from Svizera Labs Pvt. Ltd Mumbai. The Internal Standard Glipizide is purchased from Clearsynth Labs Pvt. Ltd Mumbai. Methanol (HPLC grade), Acetonitrile (HPLC grade), Formic Acid (ULC/MS), Water (Ultra Pure grade), Ascorbic Acid and Ammonium Formate were used.

\section{Instruments}

The Liquid chromatography coupled with tandem MassSpectrometer (LC-MS/MS) system consists of a Finnigan Surveyor Autosampler, Surveyor LC Pump Plus solvent delivery system and a column Oven (Thermo Electron Corporation) is used for ion separations. The Mass spectrometer was Thermo Scientific TSQ Quantum discovery max ultra triple stage quadrupole mass spectrometer used for ion detection. An Electron Spray Ionization (ESI) technique was used. Data was collected and processed using LC Quan Version 4.5.6 Data collection and integration software.

Chromatographic Condition: The Liquid Chromatographic separations were carried out by using Hypersil, Gold, 4.6 X $50 \mathrm{~mm}, 5 \mu$ Thermo. Column temperature was held at $30^{\circ} \mathrm{C}$. The auto sampler tray temperature was $10^{\circ} \mathrm{C}$. Mobile phase with a composition of Methanol: $0.1 \%$ FA in $10 \mathrm{mM}$ Ammonium Formate (90:10) v/v is pumped by the solvent delivery system at a flow rate of $0.400 \mathrm{~mL} / \mathrm{min}$.A typical injection volume was $10.0 \mu \mathrm{L}$.

MS/MS detection: Precursor ions for analyte and internal standard were determined from mass spectra obtained by the TSQ mass spectrometer. TSQ mass Spectrometer includes an electronicallycontrolled, integrated syringe pump. The Mass Spectrometric (MS) conditions for Pyrazinamide and Glipizide were optimized by separate infusion into the MS at a flow rate of $5 \mu \mathrm{L} / \mathrm{min}$ constantly, while adjusting MS parameters to achieve maximal intensity. Electro-spray

*Corresponding author: Chaitanya Krishna A, Study Director, Bombay Bioresearch Centre, Govandi-400043, Mumbai, India, E-mail: chaitanya@bbrc-cro.com

Received February 18, 2012; Accepted April 04, 2012; Published April 08, 2012

Citation: Chaitanya Krishna A, Saravanan RS, Jeevanantham S, Vignesh $R$ Karthik P (2012) Determination of Pyrazinamide in Human Plasma Samples Containing Fixed Dose Combination Molecules by using Liquid Chromatography Tandem Mass Spectrometry. Adv Pharmacoepidem Drug Safety 1:10[. doi:10.4172/

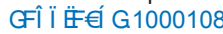

Copyright: (c) 2012 Chaitanya Krishna A, et al. This is an open-access article distributed under the terms of the Creative Commons Attribution License, which permits unrestricted use, distribution, and reproduction in any medium, provided the original author and source are credited. 
Citation: Chaitanya Krishna A, Saravanan RS, Jeevanantham S, Vignesh R, Karthik P (2012) Determination of Pyrazinamide in Human Plasma Samples Containing Fixed Dose Combination Molecules by using Liquid Chromatography Tandem Mass Spectrometry. Adv Pharmacoepidem Drug

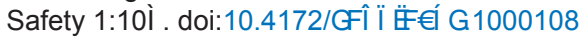

Page 2 of 5

ionization in positive ion mode (ESI+) was used for ionization and selective reaction monitoring (SRM) mode was chosen for detection. The optimized precursor ions pairs were $\mathrm{m} / \mathrm{z} 124.100 \rightarrow 79.160$ for Pyrazinamide and m/z 446.200 $\rightarrow 321.200$ for Glipizide Shown Figure 1 and Figure 2. The optimized MS parameters were as follows: Ion Spray voltage: 5000 volt, Sheath gas pressure: 50 psi, Auxiliary gas pressure: 15 psi, Capillary temperature: $265^{\circ} \mathrm{C}$. Collision Pressure: 1.5 psi. Peak areas were automatically integrated using LC Quan Version 4.5.6 (Thermo Corporation).

\section{Preparation of Calibration standards and quality control samples}

The calibration standards and the quality control (QC) samples were prepared from separate stock standard solutions. The concentrations of stock solutions were $3974.193 \mu \mathrm{g} / \mathrm{mL}$ and $3973.836 \mu \mathrm{g} / \mathrm{mL}$ for calibration standard and quality control samples. Methanol was used as diluent for preparation of the stock solutions. Spiking solutions for calibration standards and quality control concentrations were prepared in methanol: water $(80: 20) \% \mathrm{v} / \mathrm{v}$. Calibration standards were prepared by spiking corresponding solutions into blank human plasma (containing $0.1 \%$ Ascorbic acid) $[3,4]$ to provide concentrations ranging between $0.935 \mu \mathrm{g} / \mathrm{mL}$ (Lower Limit of Quantification-LLOQ) and $60.408 \mu \mathrm{g} /$ $\mathrm{mL}$ (upper limit of quantification-ULOQ). For Quality control plasma samples preparation, the corresponding spiking solutions is spiked to the human blank plasma to attain the four Quality Control (QC)

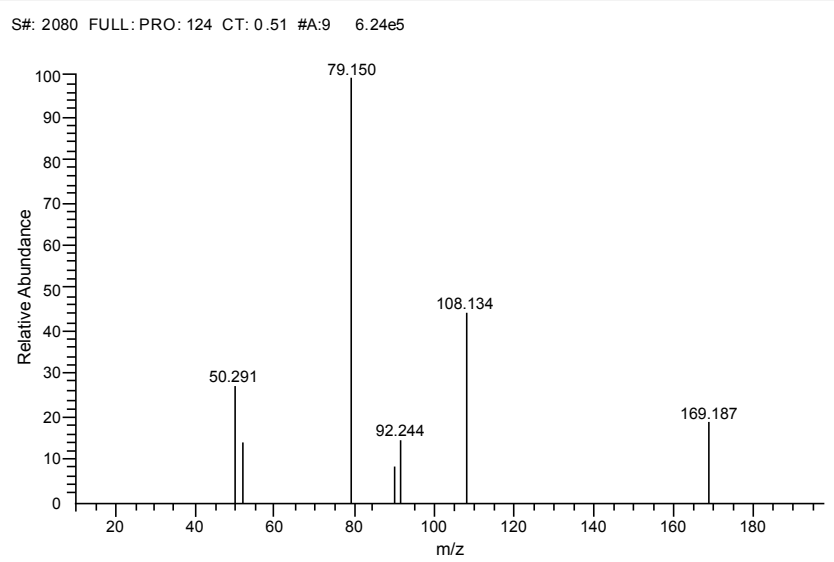

Figure 1: Product ion of Pyrazinamide.

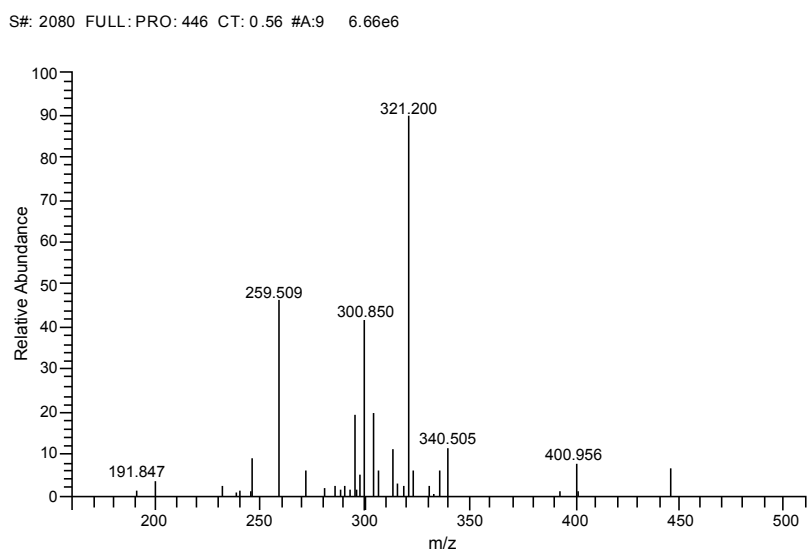

Figure 2: Product ion of Glipizide. concentrations: $0.960 \mu \mathrm{g} / \mathrm{mL}, 2.865 \mu \mathrm{g} / \mathrm{mL}, 23.386 \mu \mathrm{g} / \mathrm{mL}$ and 43.712 $\mu \mathrm{g} / \mathrm{mL}$ for achieving LOQQC (limit of Quantification QC), LQC (Low QC), MQC (Middle QC) and HQC (High QC) levels respectively. For preparation of the Calibration curve samples and Quality Control samples, the limits followed were as follows:

LLOQ - atleast equal to or less than 5 half lifes of the drug

ULOQ - two times of the Cmax of drug

LOQ QC - 100 to $105 \%$ of LLOQ

LQC -2.5 to 3 times of LLOQ

MQC - $\pm 2 \%$ of Average between LQC and HQC

HQC - $65-85 \%$ of ULOQ

For preparation of the plasma samples, volume of $20.000 \mu \mathrm{L}$ of the spiking solutions was spiked into $0.980 \mathrm{~mL}$ of human blank plasma. Internal standard stock solution $(1003.915 \mu \mathrm{g} / \mathrm{mL})$ of Glipizide was prepared in methanol and used as an Internal Standard dilution. Working solutions for internal standard $(1.506 \mu \mathrm{g} / \mathrm{mL})$ was prepared in Methanol: water (80:20) \%v/v.

\section{Sample extraction}

$200.0 \mu \mathrm{L}$ aliquot of plasma samples was mixed with $50.0 \mu \mathrm{L}$ of internal standard working solution $(1.506 \mu \mathrm{g} / \mathrm{mL})$. Pre-treatment is performed by adding $0.400 \mathrm{~mL}$ of $0.1 \%$ Formic acid and mixed the samples for approximately 10 secs on a vortex shaker before adapting the SPE procedure. Commercially available cartridge (Analchem PLEXUS $30 \mathrm{mg} / 1 \mathrm{cc}$ ) was utilized for extraction. Conditioning and equilibration of the cartridges were performed with $1 \mathrm{~mL}$ of methanol and water respectively. Pyrazinamide and Glipizide were extracted into the cartridge by loading the pre-treated plasma samples. Cartridges were washed using $2 \times 0.500 \mathrm{~mL}$ of Ultrapure Water, in order to remove the unbound substance in the cartridge and to reduce any interfering band in chromatograms. Finally $0.400 \mathrm{~mL}$ of mobile phase was used to elute Pyrazinamide. $10.0 \mu \mathrm{L}$ of the eluent was injected for chromatographic analysis.

\section{Validation [5]}

Selectivity and specificity: Blank human plasma from eight different lots (including one Haemolysed and one Lipimic) were processed without Analyte and internal standard, and with the same eight lots LLOQ level is processed to evaluate the presence of any interference at the retention time of Analyte and Internal standard.

Matrix factor: Matrix factor was evaluated at low and high quality control concentrations, to ensure that the precision, selectivity and sensitivity are not compromised due to a change in matrix. Matrix factor can be termed as the quantitative measurement of the matrix effect. Aqueous mixtures of internal standard and analyte, representing $100 \%$ extraction of internal standard and analyte, at low and high QC concentrations were prepared. These shall serve as Reference Samples. 8 different lots of blank matrices (from eight individuals, including, one Haemolysed and one Lipimic) were processed in duplicate without addition of IS. Eluted solution volumes were equally diluted with reference sample; it is compared with respective aqueous reference sample equally diluted with mobile phase.

Calibration curve and linearity: The eight-point calibration curve was constructed by plotting, peak area ratio of Pyrazinamide to their corresponding internal standard versus Pyrazinamide concentrations. A linear regression with weighing factor of linear $1 / \mathrm{x}^{2}$ was applied. 
Citation: Chaitanya Krishna A, Saravanan RS, Jeevanantham S, Vignesh R, Karthik P (2012) Determination of Pyrazinamide in Human Plasma Samples Containing Fixed Dose Combination Molecules by using Liquid Chromatography Tandem Mass Spectrometry. Adv Pharmacoepidem Drug

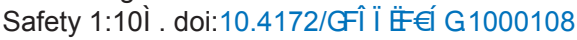

Page 3 of 5

Intra and inter-day assay accuracy and precision: Intra-day precision and accuracy were determined by analysis of six replicates of each QC sample $(n=6)$ at LOQQC, LQC, MQC and HQC concentration levels, extracted with a set of standards in one batch. Inter-day precision and accuracy was performed in two different days.

Recovery: Recovery is carried out to evaluate the loss of drugs and/ or internal standards during sample extraction. Peak area counts of drugs and internal standards from extracted QC samples were compared with corresponding QCs reference samples to evaluate any loss of either drugs or internal standards. It is preferable to observe consistent recovery for all three QC levels. For calculating the \% recovery, 6 sets of plasma samples at LQC, MQC and HQC levels were prepared, extracted and injected. Simultaneously, aqueous spiked recovery samples at LQC, MQC and HQC concentrations were prepared in singlet and injected 6 times (reference sample).

Stability: Stability of both drugs in different matrices and under different conditions was evaluated. The detailed tests are described below. Stability was assessed by comparing the mean concentration of the stored QC samples with the mean concentration of freshly prepared QC samples. Drug stability in pooled human blank plasma is a function of the storage conditions, the chemical properties of the drug and the matrix effect. The following tests were performed to evaluate the stability, Short-term and Long-term Stock solution stability, Bench top stability, Freeze and thaw stability, Autosampler stability, Wet Extract Stability, Long Term stability In Matrix.

\section{Result and Discussion}

\section{Method development}

In this method development for determination of Pyrazinamide in human plasma, some of the issues were being addressed. As the molecular weight of Pyrazinamide is low, it would be more difficult to get a good chromatographic condition, where, noise levels were found to be more in several compositions of the mobile phase and affect the sensitivity of the analysis. Finally better results were obtained by optimizing the chromatographic conditions with Methanol: $0.1 \%$ FA in $10 \mathrm{mM}$ Ammonium Formate (90:10) v/v as mobile phase. Separation was performed in Hypersil Gold Column, 4.6 X $50 \mathrm{~mm}, 5 \mu$ at a flow rate of $0.400 \mathrm{~mL} / \mathrm{min}$

Further, a method that can be used for analysis of Pyrazinamide alone is not the only alternative. Because most of the times, Bioequivalence studies are conducted with Fixed Dose Combination drugs. In this context, an analyst should be able to develop a method that can be used for the plasma samples containing all fixed dose combinations. Though the task is multiple, some more criticalities need to be considered by the researchers while developing a method for fixed dose combination molecules. Stability of the molecules is the primary concern, which will change the actual methodology for analysis of Fixed Dose Combination Drugs. For instance, a method for analysis of fixed dose combination formulation of Anti-TB product containing Isoniazid, Rifampicin, Ethambutol and Pyrazinamide will be different from the methodology and different instruments were used for the analysis of samples containing Pyrazinamide alone [5-9]. The main reason for this is being the stability concern of rifampicin $[10,3,4]$. Due to the Oxidative degradation of Rifampicin, it is better suggested that the plasma sample should contain ascorbic acid to inhibit the oxidative degradation. In this condition, a method that can be used for analysis of Pyrazinamide in Human Plasma containing ascorbic acid will be more beneficial. Previous methods used for the determination of Pyrazinamide by High Performance Liquid Chromatography (HPLC) $[6,11,7,8]$ has been found to consume more run times because of the lengthy column used for the separation, where they end up in longer time for analysis and also with animal plasma [12]. Some of the methods in LCMS/MS [11] are more tedious to perform because of the chromatographic conditions and sample preparation steps that have been adapted. More over, the new methods have to offer the benefits of cost effectiveness, less run times and simplicity in the process, with the recent advances in the analytical techniques. Recovery of the drug and IS were found to be more (60\%) when compared with the previous methods. This method is found to be simple, precise, and compatible, less time consuming for the determination of Pyrazinamide in human plasma by LCMS/MS. Authors propose a simple method that would compensate all the above mentioned problems including the stability issues.

\section{Validation}

Selectivity and specificity: No interference from the blank plasma at the retention time of the Analyte and Internal standard.

Matrix Factor: Observed \% CV of matrix factor is $8.66 \%$ and $5.50 \%$ in LQC, $3.38 \%$ and $1.80 \%$ in HQC for Pyrazinamide and Internal standard respectively. All eight matrix lots showed very similar matrix effect for both analyte and their corresponding internal standard.

Calibration Standard and Linearity: For three consecutive batches, the calibration curves showed an overall accuracy of $90.43 \%$ $113.02 \%$ with \%CV of $0.62 \%-9.86 \%$. The calibration Standard Linearity has a regression Coefficient of $>0.9929$. The detailed results are shown in Table 1. The Calibration Standards meets the acceptance criteria Chromatograms are shown from Figure 3 to Figure 9.

Accuracy and precision: Table 2 shows inter and intra assay precision and accuracy. The method was found to be highly accurate and precise. For Pyrazinamide, accuracy of $86.27 \%-105.91 \%$ and precision of $1.59 \%-8.96 \%$ for intra-assay, and accuracy of $89.17 \%-103.41 \%$ and precision of 3.04\%-8.82\% CV for inter-assay were obtained for all QC levels including LOQQC.

\begin{tabular}{|l|l|l|l|l|l|l|l|l|}
\hline \multirow{2}{*}{ Batch ID } & \multicolumn{7}{|l|}{ Theoretical concentration $(\mu \mathrm{g} / \mathrm{mL})$} \\
\cline { 2 - 10 } & 0.935 & 1.869 & 4.673 & 11.681 & 23.363 & 35.943 & 51.347 & 60.408 \\
\hline Batch 01 & 0.916 & 1.92 & 5.633 & 12.798 & 23.459 & 36.463 & 50.538 & 54.018 \\
\hline Batch 02 & 0.848 & 2.12 & 5.232 & 12.594 & 23.708 & 34.034 & 48.702 & 51.418 \\
\hline Batch 03 & 0.952 & 1.74 & 4.98 & 12.214 & 23.72 & 35.455 & 49.804 & 58.451 \\
\hline Mean & 0.905 & 1.927 & 5.282 & 12.535 & 23.629 & 35.317 & 49.681 & 54.629 \\
\hline Precision & 5.83 & 9.87 & 6.24 & 2.36 & 0.62 & 3.46 & 1.86 & 6.51 \\
\hline Accuracy & 96.83 & 103.09 & 113.03 & 107.31 & 101.14 & 98.26 & 96.76 & 90.43 \\
\hline
\end{tabular}

Table 1: Accuracy and Precision for Calibration Standards.
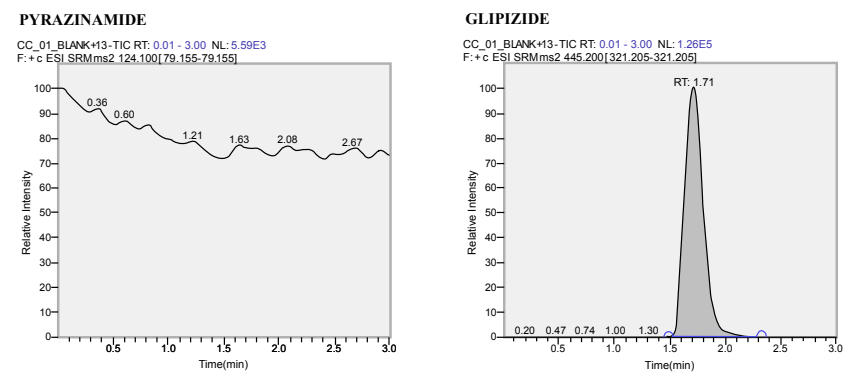

Figure 3: Blank + IS 

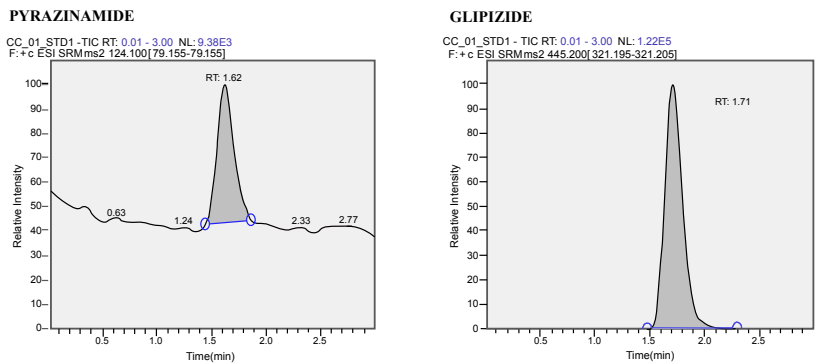

Figure 4: Standard 1.
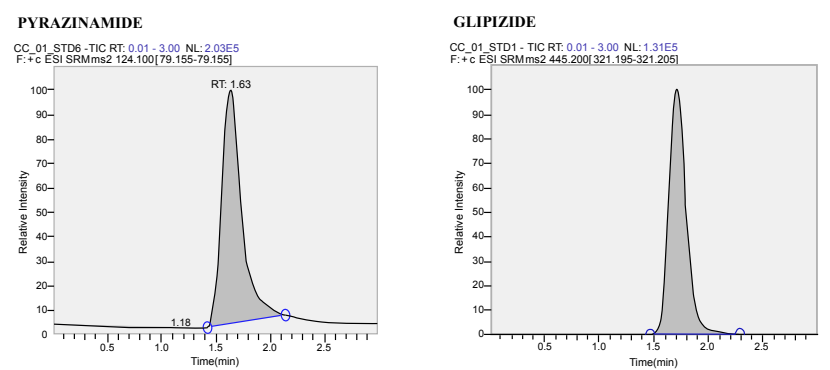

Figure 5: Standard 2.
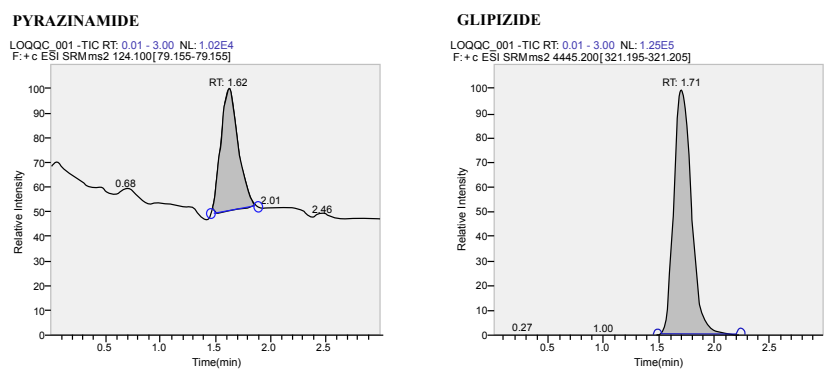

Figure 6: LOQQC
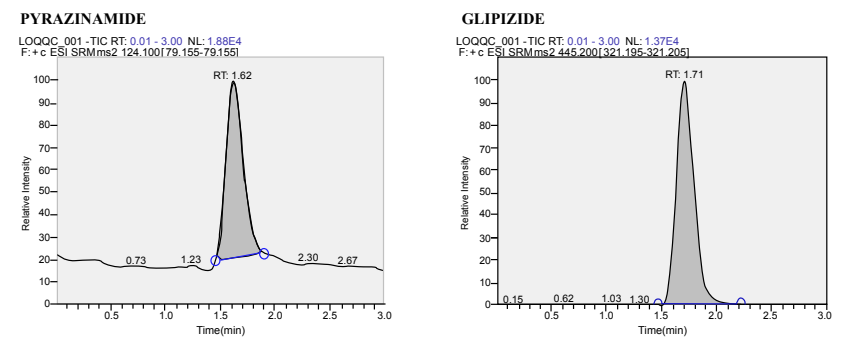

Figure 7: LQC

Recovery: Recovery was performed by processing six samples in each level of concentration and compared the area obtained with the area of reference sample (prepared in aqueous), the mean recovery is calculated. The higher mean recovery represents the effectiveness of the extraction techniques. Table 3 show the overall recovery of $61.83 \%$ for Pyrazinamide, and 59.32\% for Glipizide were obtained. Both compounds show consistent recovery results for all three QC levels.

Stability: Stability of Pyrazinamide in human plasma under differ-
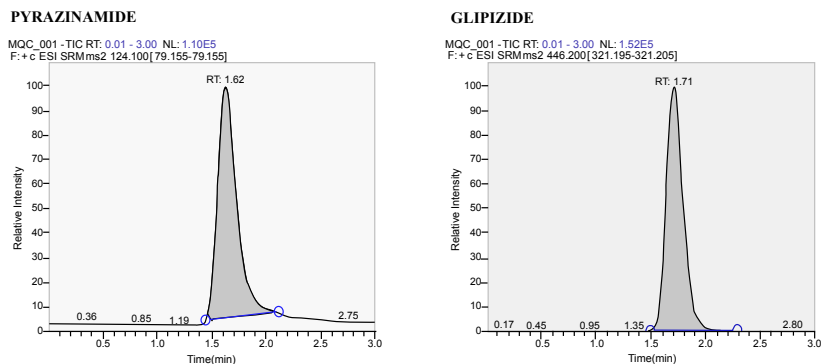

Figure 8: MQC.
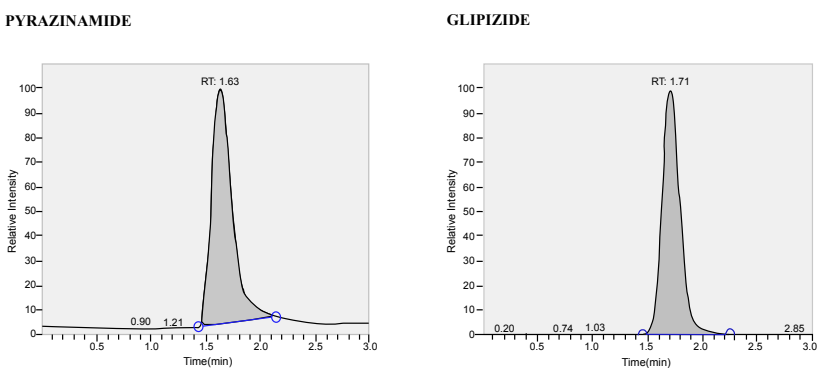

Figure 9: HQC.

\begin{tabular}{|l|l|l|}
\hline Parameter & Intra-Batch & Inter-Batch \\
\hline Accuracy (\%) & $86.27-105.91$ & $89.17-103.41$ \\
\hline Precision (\%) & $1.59-8.96$ & $3.04-8.82$ \\
\hline
\end{tabular}

Table 2: Intra and Inter-day Accuracy and Precision.

\begin{tabular}{|l|l|l|}
\hline \multirow{2}{*}{ QC Level } & Recovery (\%) & \multicolumn{2}{l|}{} \\
\cline { 2 - 3 } & Pyrazinamide & Glipizide \\
\hline LQC & 59.80 & 55.65 \\
\hline MQC & 59.64 & 58.48 \\
\hline HQC & 66.07 & 63.82 \\
\hline Recovery & $\mathbf{6 1 . 8 3}$ & $\mathbf{5 9 . 3 2}$ \\
\hline \%CV & $\mathbf{3 . 6 6}$ & $\mathbf{4 . 1 6}$ \\
\hline
\end{tabular}

Table 3: Recovery of Analyte and IS.

\begin{tabular}{|l|l|l|l|l|l|l|l|l|l|l|l|}
\hline $\begin{array}{l}\text { Param- } \\
\text { eter }\end{array}$ & $\begin{array}{l}\text { Bench Top } \\
\text { Stability }\end{array}$ & $\begin{array}{l}\text { Auto } \\
\text { Sampler } \\
\text { Stability }\end{array}$ & \multicolumn{2}{l|l|l|l|l|l|}{$\begin{array}{l}\text { Wet extract } \\
\text { stability }\end{array}$} & $\begin{array}{l}\text { Freeze } \\
\text { and Thaw } \\
\text { stability }\end{array}$ & \multicolumn{2}{l}{$\begin{array}{l}\text { Long term Ma- } \\
\text { trix stability }\end{array}$} \\
\hline $\begin{array}{l}\text { QC } \\
\text { Levels }\end{array}$ & LQC & HQC & LQC & HQC & LQC & HQC & LQC & HQC & LQC & HQC \\
\hline $\begin{array}{l}\text { Preci- } \\
\text { sion }\end{array}$ & 2.37 & 2.64 & 2.17 & 2.61 & 2 & 2.64 & 2.56 & 2.62 & 2.8 & 4.67 \\
\hline $\begin{array}{l}\% \text { Sta- } \\
\text { bility }\end{array}$ & 95.43 & 93.19 & 98.17 & 94.34 & 99.16 & 93.26 & 97.97 & 93.87 & 1.0633 & 0.9695 \\
\hline
\end{tabular}

Table 4: Stability of Pyrazinamide.

\begin{tabular}{|l|l|l|l|l|}
\hline Stability & \multicolumn{2}{|l|}{$\begin{array}{l}\text { Short term Stock Solution } \\
\text { Stability for 7 hrs }\end{array}$} & \multicolumn{2}{l|}{$\begin{array}{l}\text { Long term Stock solution } \\
\text { stability for 15days }\end{array}$} \\
\hline Molecule & Analyte & IS & Analyte & IS \\
\hline \%CV & 3.01 & 3.15 & 3.81 & 2.24 \\
\hline \% Stability & $99.84 \%$ & $100.39 \%$ & $93.93 \%$ & $101.98 \%$ \\
\hline
\end{tabular}

Table 5: Stability of Pyrazinamide.

ent conditions was evaluated. The detailed results are shown in Table 4 and Table 5, as seen from the table, three freeze/thaw cycles, $6 \mathrm{~h}$ room temperature storage, and $28 \mathrm{~h}$ autosampler stability has been estab- 
Citation: Chaitanya Krishna A, Saravanan RS, Jeevanantham S, Vignesh R, Karthik P (2012) Determination of Pyrazinamide in Human Plasma Samples Containing Fixed Dose Combination Molecules by using Liquid Chromatography Tandem Mass Spectrometry. Adv Pharmacoepidem Drug

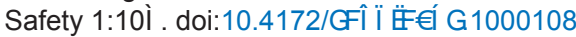

Page 5 of 5

lished. In addition wet extract stability shown for 28 hrs were established. All of these demonstrate the ruggedness of the method.

\section{Conclusion}

A simple, rapid, selective method in chromatographic condition and extraction is more precise, sensitive and also compatible for the analysis of Pyrazinamide in human plasma for fixed dose combination drugs with good recovery by using LCMS/MS. The method was developed and validated, which was found to have good in stability. In addition, this method has been used for the determination of Pyrazinamide in human plasma by LCMS/MS for four drug fixed dose combination formulation while conducting the $\mathrm{BA}$ and $\mathrm{BE}$ studies. Anyway, combination treatment with Rifampicin and Pyrazinamide is accepted as an alternative for Isoniazid therapy. Combination therapy for 2 months offers an equivalent efficacy as 12 months therapy of Isoniazid [13]. Because of these reasons, the FDC formulations are gaining main importance in the therapeutic area of tuberculosis. Hence forth, researchers proposed a method that will be more beneficial for both individual and combinational determination of Pyrazinamide in human plasma by LCMS/MS.

\section{Acknowledgements}

We are thankful to Mrs. Gauri Sapte, Director, BBRC, Dr.V.P.Shedbalkar and the management of Maneesh Pharmaceuticals Ltd., for their valuable support and contribution in carrying out this research work with out which it will not be possible to publish the paper.

\section{References}

1. (2001) The merck index.(13th edn).

2. Treatment of Tuberculosis Guidelines, fourth edition, WHO.

3. Shishoo CJ, Shah SA, Rathod IS, Savale SS, Vora MJ (2001) Impaired bioavailability of rifampicin in presence of Isoniazid from fixed dose combination (FDC) formulation. Int J Pharm 228: 53-67.

4. Swart KJ, Papgis M (1992) Automated high-performance liquid chromatographic method for the determination of rifampicin in plasma. J Chromatogr 593: 21-24.
5. U.S. Department of Health and Human Services Food and Drug Administration Center for Drug Evaluation and Research (CDER) Center for Veterinary Medicine (CVM) BP, (May 2001) Guidelines for Industry Bioanalytical Method Validation

6. Agrawal S, Singh I, Kaur KJ, Bhade SR, Kaul CL, et al. (2004) Comparative bioavailability of rifampicin, isoniazid and Pyrazinamide from a four drug fixed dose combination with separate formulations at the same dose levels. Int $\mathrm{J}$ Pharm 276: 41-49.

7. Zhou Z, Chen L, Liu P, Shen M, Zou F (2010) Simultaneous Determination of Isoniazid, Pyrazinamide, Rifampicin and Acetyl Isoniazid in Human Plasma by High-Performance Liquid Chromatography. Anal Sci 26: 1133-1138.

8. Panchagnula R, Sood A, Sharda N, Kaur K, Kaul CL (1999) Determination of rifampicin and its main metabolite in plasma and urine in presence of Pyrazinamide and Isoniazid by HPLC method. J Pharm Biomed Anal 18: 1013 1020.

9. James, DT., Suresh, K., Ramesh, N., Sasi, jSL., (2011) Method development and validation of Pyrazinamide in human plasma by using gabapentin as internal standard: Icms/ms. International journal of institutional pharmacy and life sciences $1(1)$.

10. Agrawal S, Singh I, Kanwal Jit K, Bhade S, Chaman L K, et al. (2004) Bioequivalence trials of rifampicin containing formulations: extrinsic and intrinsic factors in the absorption of Rifampicin. Pharmacological Research 50: 317-327.

11. Gong Z, Basir Y, Chu D, McCort-Tipton M (2009) A rapid and robust liquid chromatography/tandem mass spectrometry method for simultaneous analysis of anti-tuberculosis drug-Ethambutol and Pyrazinamide in human plasma. J Chromatogr B Analyt Technol Biomed Life Sci 877: 1698-1704.

12. Kaka JS (2006) Rapid Simplified Analysis of Pyrazinamide in Rat Plasma by HPLC. Journal of Liquid Chromatography 17: 3793-3801.

13. Gordin F, Chaisson RE, Matts JP, Miller C, de Lourdes Garcia M, et al (2000) Rifampin and pyrazinamide vs isoniazid for prevention of tuberculosis in HIVinfected persons: an international randomized trial. Terry Beirn Community Programs for Clinical Research on AIDS, the Adult AIDS Clinical Trials Group, the Pan American Health Organization, and the Centers for Disease Control and Prevention Study Group. JAMA 283: 1445-1450. 